\title{
Re-evaluation of single nucleotide variants and identification of structural variants in a cohort of 45 sudden unexplained death cases
}

\author{
Jacqueline Neubauer ${ }^{1}$ (1) $\cdot$ Shouyu Wang ${ }^{1,2} \cdot$ Giancarlo Russo $^{3}$ (D) Cordula Haas $^{1}$ (i)
}

Received: 2 January 2021 / Accepted: 16 March 2021 / Published online: 25 April 2021

(c) The Author(s) 2021

\begin{abstract}
Sudden unexplained death (SUD) takes up a considerable part in overall sudden death cases, especially in adolescents and young adults. During the past decade, many channelopathy- and cardiomyopathy-associated single nucleotide variants (SNVs) have been identified in SUD studies by means of postmortem molecular autopsy, yet the number of cases that remain inconclusive is still high. Recent studies had suggested that structural variants (SVs) might play an important role in SUD, but there is no consensus on the impact of SVs on inherited cardiac diseases. In this study, we searched for potentially pathogenic SVs in 244 genes associated with cardiac diseases. Whole-exome sequencing and appropriate data analysis were performed in 45 SUD cases. Re-analysis of the exome data according to the current ACMG guidelines identified 14 pathogenic or likely pathogenic variants in 10 (22.2\%) out of the 45 SUD cases, whereof $2(4.4 \%)$ individuals had variants with likely functional effects in the channelopathy-associated genes SCN5A and TRDN and $1(2.2 \%)$ individual in the cardiomyopathy-associated gene DTNA. In addition, 18 structural variants (SVs) were identified in 15 out of the 45 individuals. Two SVs with likely functional impairment were found in the coding regions of PDSS2 and TRPM4 in 2 SUD cases (4.4\%). Both were identified as heterozygous deletions, which were confirmed by multiplex ligation-dependent probe amplification. In conclusion, our findings support that SVs could contribute to the pathology of the sudden death event in some of the cases and therefore should be investigated on a routine basis in suspected SUD cases.
\end{abstract}

Keywords Forensics · Massively parallel sequencing (MPS) · Exome sequencing · Single nucleotide variant (SNV) . Structural variants $(\mathrm{SV}) \cdot$ Copy number variation $(\mathrm{CNV})$

\section{Introduction}

Sudden death events in young individuals often represent the first manifestation of an undetected genetic disease, which remained without any symptoms during lifetime. Although some of the deceased show cardiac structural

Jacqueline Neubauer and Shouyu Wang contributed equally to this work.

Jacqueline Neubauer

jacqueline.neubauer@irm.uzh.ch

1 Zurich Institute of Forensic Medicine, University of Zurich, Zurich, Switzerland

2 Department of Forensic Medicine, School of Basic Medical Sciences, Fudan University, Shanghai, China

3 Functional Genomics Center Zurich (FGCZ), University of Zurich/ETH, Zurich, Switzerland abnormalities and can be explained by autopsy, approximately $30 \%$ of sudden death cases still remain elusive and are therefore termed as sudden unexplained death (SUD) $[1,2]$. In the past years, postmortem molecular autopsy enabled the identification of hidden cardiac diseases in a series of SUD cases. These diseases include mainly channelopathies or cardiomyopathies associated with single nucleotide variants (SNVs) located in the coding regions of functional genes [3-6]. In addition, some variants located in noncoding regions, such as regulatory elements and splice sites, might be accountable for serious functional effects [7-10]. Recent studies revealed that structural variants (SVs), including copy number variations (CNVs), might play an important role in SUD and could explain some of the unresolved cases [11-14].

SVs include diverse genomic alterations like deletions, duplications, insertions, inversions, translocations, or complex rearrangements of relatively large segments such as 
tandem exon duplications, or complex gains or losses of homologues sequences at multiple sites in the genome, commonly referred to as CNVs $[15,16]$. While SVs are considerably less common than SNVs, they have greater functional potential due to their larger size, and they are more likely to alter gene structure [17]. SVs and CNVs can influence gene expression, phenotypic variation, and adaptation, by disrupting genes and altering gene dosage. Many SVs are described to confer risk to complex disease traits or increase disease susceptibility to e.g. HIV-1 infection [18] or neurodevelopmental disorders including autism [19], schizophrenia [20], and depression [21]. Besides, pathogenic CNVs have been reported in cardiac diseases such as Brugada syndrome (BrS) [11], arrhythmogenic right ventricular cardiomyopathy (ARVC) [12], or sudden cardiac death (SCD)-related cases $[13,14]$. One previous study examined CNVs in a large cohort of SUD cases and patients that had an inherited cardiac disease and discovered that the frequency of identified CNVs varied from 1.4 to $5.1 \%$ among cases with different underlying diseases [22]. Another study investigated a small cohort of 13 sudden arrhythmic death syndrome (SADS) and sudden unexplained death in infancy (SUDI) cases with whole genome sequencing and whole transcriptome sequencing. However, they did not find any SVs in the coding and regulatory regions of 100 cardiac genes [23]. A recent study focused on 2 large Amish families with multiple sudden deaths and sudden cardiac arrests in young individuals and identified a homozygous multi-exon duplication in $R Y R 2$ [24]. Another study investigated a Tunisian family with high incidence of SCD in young family members, but did not find any pathological relevant CNVs [25]. The lack of a consensus of the impact of SVs on inherited cardiac diseases emphasized the need of more evidence on this topic.

The aim of this study was to screen for potentially pathogenic SVs in 244 genes associated with cardiac diseases in a cohort of 45 SUD cases. Whole-exome sequencing (WES) in 35 out of these 45 cases has already been performed earlier [4], whereas 10 additional SUD cases were analyzed as part of this study. Due to updates in the recommendations for rare variant classification, the exome sequencing data of all 45 SUD cases was analyzed and classified according to the current ACMG guidelines [26].

\section{Materials and methods}

\section{SUD cohort}

All SUD cases had been autopsied at the Zurich Institute of Forensic Medicine (ZIFM) in Switzerland between 2012 and 2019 . The sudden death victims were examined according to the respective European and Swiss guidelines for the management of young SUD cases [27-29], including a thorough death scene investigation, a complete autopsy with pharmacological-toxicological and histopathological screening, and a review of the clinical history. The SUD cohort consisted of 45 cases with a mean age of $30.2 \pm 14.5$ years (range: 1-63 years of age) (Table S1). Seventy-five percent of the deceased were males and most of them were of European origin (89\%). Another 5 cases (11.1\%) were of African (3 cases), Indian (1 case), and Chinese (1 case) origin. Exome sequencing data was already available for 35 of the SUD cases [4]. Additional 10 SUD cases were analyzed according to the workflow described in our previous study [4].

\section{Exome sequencing and bioinformatics}

Genomic DNA of the 10 new SUD cases was obtained from shock-frozen kidney tissue. DNA extraction was performed using the QIAamp DNA Mini Kit (Qiagen, Hombrechtikon, Switzerland) according to the manufacturer's protocol. DNA quantities were determined with a Quantus ${ }^{\mathrm{TM}}$ Fluorometer (Promega, Dübendorf, Switzerland). The SureSelect ${ }^{\mathrm{XT}} \operatorname{tar}^{\mathrm{H}}$ get enrichment and SureSelect ${ }^{\mathrm{XT}}$ All Exon V5 + UTR kits (Agilent Technologies AG, Basel, Switzerland) were used for DNA library preparation and exome capture, followed by quality and quantity assessment with the TapeStation system using DNA 1000 ScreenTapes (Agilent Technologies AG). DNA libraries were sequenced on an Illumina NovaSeq 6000 instrument (Illumina Inc., San Diego, CA, USA) with 150 paired-end reads and a mean coverage of $65 \times$ per sample. Sequences were aligned to the reference genome (GRCh37/ hg19) using BWA [30] and samples were required to have at least $80 \%$ of the exome covered at $\geq 20 \times$ read depth. Variant discovery was performed by means of GATK [31], following the GATK best practice workflow [32].

\section{SNV identification}

Data analysis was confined to a target gene panel consisting of 244 genes associated with cardiac diseases (Table S2). The annotation of the VCF files was performed with the software Alamut Batch, version 1.11 (Interactive Biosoftware, Rouen, France) and the visualization of the coverage of SNVs was done with the software IGV, version 2.4.16 [33]. SNV screening was performed according to an updated version of our previously published in-house filtering strategy [4] and to the ACMG standards and guidelines for the interpretation of sequence variants [26]. Our filter strategy selects SNVs with a stringent global minor allele frequency value (MAF) of less than or equal to $0.005 \%$ derived from the genome aggregation database (gnomAD) which is the largest available human database, including 125,748 exomes and 15,708 whole genomes from unrelated individuals of different ethnicities, sequenced as part of various population genetic studies [34]. In addition, synonymous and intronic 
variants were excluded, focusing only on exonic and splice site SNVs. Pathogenicity of the SNVs was assigned based on the evaluation of variant types (null variants, frameshift variants, splice site variants, or missense variants) and on the genome interpretation database VarSome [35]. Identified SNVs have been submitted to the Leiden Open Variation Database (Individuals Nr. 00,315,499-00,315,504 and 00,324,655-00,324,658) (http://databases.lovd.nl).

\section{SV identification}

SVs were called using LUMPY [15]. Briefly, discordant paired-end alignments were retrieved using samtools [36] (samtools view $-b-F$ 1294) and the split-read alignments were extracted using the LUMPY auxiliary script extractSplitReads_BwaMem. The discordant paired-end and the split-read file were then parsed, together with the alignment (bam) file to lumpyexpress with default settings. The resulting VCF files were annotated using ANNOVAR [37] using the UCSC human genome version GRCh37/hg19 as reference and the Database of Genomic Variants (DGV) as variants database [38]. Among the SVs within our target genes, those identified in more than one sample were excluded from subsequent analysis, since pathogenic SVs that contribute to SUD should be rare variants according to the ACMG guidelines [26]. Furthermore, SVs longer than $1 \mathrm{Mbp}$ were excluded because in these cases, the expression levels of too many genes were considered abnormal, which makes them difficult to validate. The remaining SVs were manually checked using the IGV software; only those with a characteristic gene expression alteration were considered eligible SV candidates. Allele frequencies of the candidate SVs were checked in the gnomAD-SV database [45].

\section{MLPA validation}

The identified candidate SVs were confirmed by multiplex ligation-dependent probe amplification (MLPA) according to the manufacturer's instructions. Self-designed oligos for the detection of our target regions were synthesized by Microsynth (Microsynth AG, Balgach, Switzerland). In brief, 100 ng of DNA and a customized probemix consisting of synthetic oligos (Table S3) and the P200 reference probemix (MRC Holland, Amsterdam, Netherlands) were used in the hybridization reaction. Fragment separation was performed on a 3130xl Genetic Analyzer (Thermo Fisher Scientific, Reinach, Switzerland). Fragment analysis and comparative analysis results were generated using Coffalyser.Net software (MRC Holland).

\section{Results}

\section{SNV identification}

Following the current ACMG guidelines for variant interpretation [26], 14 pathogenic or likely pathogenic SNVs were identified in $10(22.2 \%)$ out of the 45 SUD cases (Table 1 and Table S4). These variants were located in genes that are linked to cardiomyopathies (1 SUD case), ion channelopathies (2 SUD cases), connective tissue diseases and/ or congenital malformation syndromes (3 SUD cases), and metabolic diseases (4 SUD cases). Out of these 14 SNVs, 2 heterozygous stop-gain variants (DTNA, NM_001390.4, c. $2224 \mathrm{C}>\mathrm{T}$, p.(Gln742*) and LZTR1, NM_006767.4, c. $2440 \mathrm{C}>\mathrm{T}$, p. $\left.\left(\mathrm{Gln} 814^{*}\right)\right), 1$ heterozygous two-base duplication (CALR3, NM_145046.4, c.387dup, p.(Ile130Tyrfs*11)), 1 homozygous one-base deletion (LZTR1, NM_006767.4, c.604_605del, p.(Met202Valfs*57)), and 1 heterozygous missense variant (SCN5A, NM_001099404.1, c. $2204 \mathrm{C}>\mathrm{A}$, p.(Ala735Glu)) were predicted as pathogenic. In addition, 2 heterozygous stop-gain variants (ALMS1, ENST00000264448.6, c.54_55insTAG, p.(Glu19*) and MLYCD, NM_012213.3, c.1073C > A, p.(Ser358*)), 2 heterozygous deletions (SLC37A4, ENST00000357590.5, c.528del, p.(Val177Trpfs*35) and TRDN, NM_006073.4, c.1923_1924del, p.(Leu643Serfs*19)), and 5 heterozygous missense variants (ACADS, NM_000017.4, c.320G > A,

Table 1 Pathogenic or likely pathogenic SNVs identified in our SUD cohort

\begin{tabular}{|c|c|c|c|c|c|c|c|c|c|c|c|c|c|}
\hline $\begin{array}{l}\text { Case } \\
\end{array}$ & rs-Nr. & Gene & HGVS genomic RelSeq-Nr. & HGVS ReISeq-Nr. & $\begin{array}{c}\text { Coding effect } \\
\end{array}$ & CDNA & $\begin{array}{l}\text { Protein change } \\
\end{array}$ & MAF (gnomAD $\left.{ }^{44}\right)$ & $\begin{array}{l}\text { Pathogenicity } \\
\end{array}$ & ACMG categories ${ }^{26}$ & Coverage & Heteroxygous allele frequency & Associated diseases \\
\hline SUDS008 & rs 137854611 & SCNSA & Chr33(GRCh37): $: 38639278 \mathrm{G}>\mathrm{T}$ & NM_001099404.1 & $\begin{array}{c}\text { missense } \\
\text { nats }\end{array}$ & $c .2204 \mathrm{C}>\mathrm{A}$ & p.(Ala735Glu) & 0.0000040140 & pathogenic & $\begin{array}{l}\text { PS3, PM1, PM2, PM5, PP2, PP3, PP5 } \\
\end{array}$ & & & BrS, LQTS \\
\hline SUDS028 & rs7767888495 & LZTRI & Chr22(GRCh37):g.21343924_21343925del & NM_006767.4 & frameshift & c.604_605del & p. (Met202Valfs *57) & 0.0000279100 & pathogenic & PVS1, PM2, PP3 & 37 & 0.47 & Noonan syndrome \\
\hline SUDS030 & & SLC37A4 & Chr1 1(GRCh37):g.118898435 del & ENST00000357590.5 & frameshift & c.528del & p.(Vall 177Trpis*35) & 0.00000000000 & likely pathogenic & PVS1, PM2, PP3 3 & 91 & homoz, & Glycogen storage disease \\
\hline SUDS038 & rs755697930 & ACADS & Chr12(GRCh37):g.121 174898G >A & NM_000017.4 & missense & c. $320 \mathrm{G} \gg \mathrm{A}$ & p.(Arg 107His) & 0.0000363100 & likely pathogenic & PMI, PM2, PM5, PP2, PP3 & 56 & 0.48 & Acyl-CoA dehydrogenase deficiency \\
\hline SUDS051 & rs539219309 & ACADS & Chr12(GRCh37):g.121176354CT & NM__000017.4 & missense & c. $814 \odot \mathrm{T}$ & p. $(\operatorname{Arg} 272 \mathrm{Cy})$ & 0.0000322600 & likely pathogenic & PM1, PM2, PP2, PP3 & 77 & 0.49 & Acyl-CoA dehydrogenase deficiency \\
\hline SUDS059 & & DTNA & Chrl 8(GRCh37):g.32464701C>T & NM_-001390.4 & stop gain & $c .2224 C \mathrm{~T}$ & p. $\left(\operatorname{Gln} 742^{\circ}\right)$ & 0.00000000000 & pathogenic & PVS1, PM2, PP3 & 562 & 0.44 & LVNC \\
\hline SUDS066 & & ALMSI & Chr2(GRCh37): 9.73613050 _7361 305 linsTAG & 1264448.6 & stop gain & c. 54 _ 55 instAG & p.(Glul & 0.0000 & likely pathogenic & PVS1, & 31 & 0.38 & Alstrom syndrome \\
\hline SUDS066 & & sost & $\mathrm{Chr} 2(\mathrm{GRCh} 37) \mathrm{g} .39233616 \mathrm{CA}$ & NM_ 005633.3 & missense & $c .2728 \mathrm{G}>\mathrm{T}$ & p.(Asp910Tyr) & 0.00000000000 & likely pathogenic & , pp2, pp3 & 45 & 0.41 & Noonan syndrome \\
\hline SUDS066 & & $F B N 2$ & $\mathrm{Chr5}$ (GRCh37):g.127674660T>C & NM_001999.4 & missense & c. $3437 \mathrm{~A}>\mathrm{G}$ & p.(Tyr1 1 146Cys) & 0.00000000000 & likely pathogenic & PY, PPS & 193 & 0.48 & Marfan syndrome \\
\hline SUDS075 & rs1 1392689787 & LZTRI & Chr22(GRCh37):g.21351554C $>\mathrm{T}$ & NM_006767.4 & stop gain & $c .2440 \mathrm{C} \gg \mathrm{T}$ & p. $\left(\mathrm{Gln} 814^{\circ}\right)$ & 0.00000000000 & pathogenic & PVS1, PM2, PP3 3 & 180 & 0.42 & Noonan syndrome \\
\hline SUDS084 & rs781420323 & TRDN & Chro(GRCh37):g.123576253_123576254del & NM_-006073.4 & frameshift & c.1923_1924del & p. (Leu643Serfs*19) & 0.0000300600 & likely pathogenic & PVSI, PM2, & 23 & 0.43 & \\
\hline SUDS112 & & CALR3 & Chr19(GRCh37):g.16601 188dup & NM_145046.4 & frameshift & c.387dup & p.(Ilel 30Tyrss"11) & 0.00000000000 & pathogenic & PVS1, PM2, PP3 & 98 & 0.45 & Familial HCM, ARVC \\
\hline SUDS11 & & MLYCD & Chr 16(GRCh37):g.83948685C $>\mathrm{A}$ & NM_012213.3. & stop gain & c. $1073 \bigcirc \mathrm{A}$ & p. (Ser358*) & 0.00000000000 & likely pathogenic & $\mathrm{S1}, \mathrm{PM} 2, \mathrm{BH}$ & 112 & 0.48 & MLYCD deficienc \\
\hline & & $A N K 2$ & $\mathrm{Chr} 4(\mathrm{GRCh} 37) \mathrm{g} .114264208 \mathrm{C}>\mathrm{G}$ & & & & he1386Leu) & & likely pathogenic & 1, PMZ, PH & & & DTS CPYT \\
\hline
\end{tabular}

ACMG American College of Medical Genetics and Genomics recommendations, ARVC arrhythmogenic right ventricular cardiomyopathy, BrS Brugada syndrome, CPVT catecholaminergic polymorphic ventricular tachycardia, gnomAD genome aggregation database, $H C M$ hypertrophic cardiomyopathy, LQTS long QT syndrome, $L V N C$ left ventricular non-compaction cardiomyopathy, NA not available

Color description for ACMG categories: red=very strong and strong evidence of pathogenicity, orange = moderate and supporting evidence of pathogenicity, green $=$ supporting evidence of benign impact 
p.(Arg107His); ACADS, NM_000017.4, c.814C > T, p.(Arg272Cys); SOS1, NM_005633.3, c.2728G > T, p.(Asp910Tyr); FBN2, NM_001999.4, c.3437A > G, p.(Tyr1146Cys) and ANK2, NM_001148.5, c.4158C > G, p.(Phe1386Leu)) were predicted as likely pathogenic.

In general, the re-analysis and re-classification of our recently published exome data of the 35 SUD cases [4] caused a change in the classification of the pathogenicity in the previously reported 11 variants (Table S5). Six of the 11 previously reported variants in the genes $A C A D 9$ (p.(Arg420Cys)), AKAP9 (p.(Asn2045Ser)), FBN2 (p.(Gln2432His)), MYLK (p.(Arg1250His)), SEMA3A (p.(Arg66Trp)), and $R Y R 2$ (p.(Glu1127Gly)) had a MAF greater than $0.005 \%$ and were therefore filtered out. The pathogenicity of the remaining 5 variants were down-classified from probably pathogenic (BMPR2 (p.(Pro864Leu)) and KCN5E (p.(Tyr62Asn))) or likely pathogenic (EFEMP2
(p.(Arg185His)), RYR2 (p.(Ala3814Val)), and $R Y R 2$ (p.(Gln4164Glu)) to uncertain significance (Table S5).

\section{SV identification}

After pre-screening and IGV check, a total of $18 \mathrm{SVs}$ were identified in 15 out of the 45 SUD cases (Table 2), located in 17 different genes (ABCC9, CDH2, DMPK, DPP6, EFEMP2, FXN, GPDIL, KCNJ2, LAMA4, NOS1AP, PDLIM3, PDSS2, PPA2, PRKAG2, PRKG1, PTPN11, TRPM4). The lengths of these SVs varied from 35 to $6079 \mathrm{bp}$, and most of them were positioned in the intergenic or intronic regions of our target genes. Out of these $18 \mathrm{SVs}$, the most promising 2 were heterozygous deletions located in the coding regions of $P D S S 2$ and TRPM4, respectively (Fig. S1).

The 71-bp deletion of PDSS2 (NM_020381.4: c.10094103_1009-4033del), which can only be identified in exon 3 of one gene isoform (transcript ID: ENST00000449027),

Table 2 SVs identified in our SUD cohort

\begin{tabular}{|c|c|c|c|c|c|c|c|c|c|}
\hline Case No. & Gene & Chrom. & Start position & End position & SV type & $\begin{array}{l}\text { SV length } \\
\text { (ABS) }\end{array}$ & Annotation & $\begin{array}{l}\text { MAF (gno- } \\
\text { mAD-SV }\end{array}$ & Pathogenicity \\
\hline SUDS006 & PRKG1 & chr10 & $53,578,486$ & $53,578,799$ & DEL & 313 & $\begin{array}{l}\text { Intronic in all gene } \\
\text { isoforms }\end{array}$ & 0.0000 & $\begin{array}{l}\text { Uncertain signifi- } \\
\text { cance }\end{array}$ \\
\hline SUDS021 & EFEMP2 & chr11 & $65,642,111$ & $65,643,519$ & DEL & 1408 & Intergenic & 0.5326 & NA \\
\hline SUDS023 & LAMA4 & chr6 & $112,430,849$ & $112,431,010$ & DEL & 161 & $\begin{array}{l}\text { Intronic in all gene } \\
\text { isoforms }\end{array}$ & 0.0000 & $\begin{array}{l}\text { Uncertain signifi- } \\
\text { cance }\end{array}$ \\
\hline SUDS028 & GPD1L & chr3 & $32,102,052$ & $32,107,883$ & DEL & 5831 & Intergenic & 0.4791 & NA \\
\hline SUDS030 & $D M P K$ & chr19 & $46,278,659$ & $46,279,615$ & DEL & 956 & $\begin{array}{l}\text { Intronic in all gene } \\
\text { isoforms }\end{array}$ & 0.3946 & $\begin{array}{l}\text { Uncertain signifi- } \\
\text { cance }\end{array}$ \\
\hline SUDS030 & PDLIM3 & chr4 & $186,441,637$ & $186,444,073$ & DEL & 2436 & $\begin{array}{l}\text { Intronic in all gene } \\
\text { isoforms }\end{array}$ & 0.0000 & NA \\
\hline SUDS033 & KCNJ2 & $\operatorname{chr} 17$ & $68,455,097$ & $68,461,176$ & DEL & 6079 & Intergenic & 0.0000 & NA \\
\hline SUDS049 & $A B C C 9$ & $\operatorname{chr} 12$ & $22,016,217$ & $22,016,262$ & DEL & 45 & $\begin{array}{l}\text { Intronic in all gene } \\
\text { isoforms }\end{array}$ & 0.0000 & $\begin{array}{l}\text { Uncertain signifi- } \\
\text { cance }\end{array}$ \\
\hline SUDS058 & PDSS2 & chr6 & $107,519,092$ & $107,519,163$ & DEL & 71 & $\begin{array}{l}\text { Coding region } \\
\text { (ENST00000449027) }\end{array}$ & 0.0075 & $\begin{array}{l}\text { Uncertain signifi- } \\
\text { cance }\end{array}$ \\
\hline SUDS067 & PPA2 & chr4 & $106,370,093$ & $106,370,417$ & DEL & 324 & $\begin{array}{l}\text { Intronic in all gene } \\
\text { isoforms }\end{array}$ & 0.0000 & $\begin{array}{l}\text { Uncertain signifi- } \\
\text { cance }\end{array}$ \\
\hline SUDS074 & PTPN11 & $\operatorname{chr} 12$ & $112,913,870$ & $112,914,196$ & DEL & 326 & $\begin{array}{l}\text { Intronic in all gene } \\
\text { isoforms }\end{array}$ & 0.0000 & $\begin{array}{l}\text { Uncertain signifi- } \\
\text { cance }\end{array}$ \\
\hline SUDS074 & PRKG1 & chr10 & $53,341,595$ & $53,341,937$ & DEL & 342 & $\begin{array}{l}\text { Intronic in all gene } \\
\text { isoforms }\end{array}$ & 0.0000 & $\begin{array}{l}\text { Uncertain signifi- } \\
\text { cance }\end{array}$ \\
\hline SUDS075 & TRPM4 & chr19 & $49,686,029$ & $49,686,064$ & DEL & 35 & $\begin{array}{l}\text { Exonic in all gene } \\
\text { isoforms }\end{array}$ & 0.0000 & Pathogenic \\
\hline SUDS075 & NOS1AP & chr1 & $162,052,680$ & $162,052,985$ & DEL & 305 & $\begin{array}{l}\text { Intronic in all gene } \\
\text { isoforms }\end{array}$ & 0.0000 & $\begin{array}{l}\text { Uncertain signifi- } \\
\text { cance }\end{array}$ \\
\hline SUDS077 & $\mathrm{CDH} 2$ & chr18 & $27,629,690$ & $27,630,006$ & DEL & 316 & Intergenic & 0.5876 & $\begin{array}{l}\text { Uncertain signifi- } \\
\text { cance }\end{array}$ \\
\hline SUDS080 & PRKAG2 & chr7 & $151,523,113$ & $151,523,386$ & DEL & 273 & $\begin{array}{l}\text { Intronic in all gene } \\
\text { isoforms }\end{array}$ & 0.0000 & $\begin{array}{l}\text { Uncertain signifi- } \\
\text { cance }\end{array}$ \\
\hline SUDS085 & DPP6 & chr7 & $154,671,008$ & $154,671,313$ & DEL & 305 & $\begin{array}{l}\text { Intronic in all gene } \\
\text { isoforms }\end{array}$ & 0.0000 & $\begin{array}{l}\text { Uncertain signifi- } \\
\text { cance }\end{array}$ \\
\hline SUDS112 & $F X N$ & chr9 & $71,665,339$ & $71,665,557$ & DEL & 218 & $\begin{array}{l}\text { Intronic in all gene } \\
\text { isoforms }\end{array}$ & 0.0000 & $\begin{array}{l}\text { Uncertain signifi- } \\
\text { cance }\end{array}$ \\
\hline
\end{tabular}

$A B S$ absolute value, $D E L$ deletion, $S V$ structural variant, $M A F$ minor allele frequency, $N A$ pathogenicity not available in the human genomics database VarSome 
was predicted to be a variant with uncertain significance. This SV was identified in an 11-year-old previously healthy girl, who was swimming in the lake when she suddenly disappeared in the water. A lifeguard observed the incident and rescued her and immediately started resuscitation. The emergency team diagnosed a ventricular fibrillation and she became defibrillated for a single event. In the children's hospital, she had a serious derailment of the acid-base balance and the sugar metabolism and died shortly after. According to the forensic investigations, the girl was already unconscious when she disappeared under water. Histological examination of the heart tissue revealed pre-existing changes in the excitation conduction system of the heart in the area of the secondary pacemaker center of the heart, which could have triggered cardiac arrhythmia and unconsciousness leading to a cardiovascular arrest.

The 35-bp deletion in exon 11 (transcript ID: ENST00000252826) of TRPM4 (NM_017636.4: c.1459_1494del, p.(Lys487_Leu498del)) was predicted as pathogenic. This SV was detected in a 38 -year-old male. He complained of discomfort after drinking some alcohol and was found dead some hours later in his bed. He did not have any medical history; however, his wife reported that he felt unusually tired and exhausted in the last 2 months prior to death. Autopsy investigation revealed an enlarged heart (520 g, 56\% enlarged according to Zeek [40]) with thickening of the heart chamber wall muscle.

\section{MLPA validation}

MLPA validation was performed on the 2 most promising SVs in the coding regions of PDSS2 and TRPM4, respectively. According to the comparative analysis results of Coffalyser.Net, both SVs were confirmed to be heterozygous deletions (Fig. 1).

\section{Discussion}

Sudden unexplained death (SUD) takes up a considerable part in overall sudden death cases, especially in adolescents and young adults. During the past decade, many channelopathy- and cardiomyopathy-associated SNVs have been identified in SUD studies by means of postmortem molecular autopsy [3-6], yet the number of cases that remain inconclusive is still high. The aim of this study was to re-analyze the exome data of 45 SUD cases according to the current ACMG guidelines and to search for potentially pathogenic SNVs and SVs.

Exome sequencing data was already available for 35 SUD cases, whereas additional 10 SUD cases were sequenced and analyzed for rare SNVs within the scope of this study. Following the current ACMG guidelines for variant interpretation [26], we re-analyzed and re-classified the SNVs in the 35 SUD cases in addition to the 10 newly sequenced SUD cases. A total of 14 pathogenic or likely pathogenic variants were identified in $10(22.2 \%)$ of the 45 SUD cases. Out of these 10 cases, 1 individual (2.2\%) carried a pathogenic variant in the cardiomyopathy-associated gene DTNA and 2 individuals (4.4\%) carried 2 (1 pathogenic and 1 likely pathogenic) variants in the channelopathy-associated genes $S C N 5 A$ and $T R D N$, respectively. Variants in the alpha-dystrobrevin encoding gene DTNA have been reported in patients with congenital heart disease and left ventricular non-compaction (LVNC) [41]. The SCN5A gene encodes the alpha subunit of the main cardiac sodium channel $\mathrm{Na}_{\mathrm{v}} 1.5$ and variants in this gene have been found to be causatively associated with BrS, long QT syndrome, cardiac conduction system dysfunction, and dilated cardiomyopathy [42]. Triadin-1, encoded by TRDN, is an important component of the calcium release unit in the sarcoplasmic reticulum of cardiac myocytes and a number of variants have been identified in patients with catecholaminergic polymorphic ventricular tachycardia (CPVT) [43].

Other studies already pointed out the importance of reanalysis of sequencing data, especially in patients with complex genetic diseases, as genomic databases are continuously updated and adjusted based on new findings in co-segregation studies and functional analyses [44]. Accordingly, when comparing our previously published data and the results presented in this study, some variants were interpreted differently and some new variants popped up. These changes are caused by the updated candidate gene list, a more stringent MAF as recommend by Tester et al. [45] and the assessment of pathogenicity based on the genome interpretation database VarSome that includes additional interpretation criteria, such as if the variant is located in a mutational hot spot and/or critical and well-established functional domain [35]. Nevertheless, many variants remain with uncertain significance emphasizing the importance of cautious interpretation, especially in cases without a clear phenotype and a complex genetic contribution. In addition, co-segregation analysis and functional assays are recommended to further evaluate the pathogenicity of identified variants.

The focus of this study was a genetic screening for SVs as potential contribution to the sudden unexpected death event. A total of $18 \mathrm{SVs}$ were identified in 15 out of the 45 individuals, but only $2(11.1 \%)$ were located on exons. The 2 exonic SVs were confirmed by MLPA as heterozygous deletions. The functional annotation of the $2 \mathrm{SVs}$ was checked in several databases.

The 71-bp heterozygous deletion in PDSS2 was previously identified as nsv4140011 by whole genome sequencing (WGS) in the gnomAD structural variants study [39]. However, neither validation information nor clinical assertion has been reported for this SV. According to the 

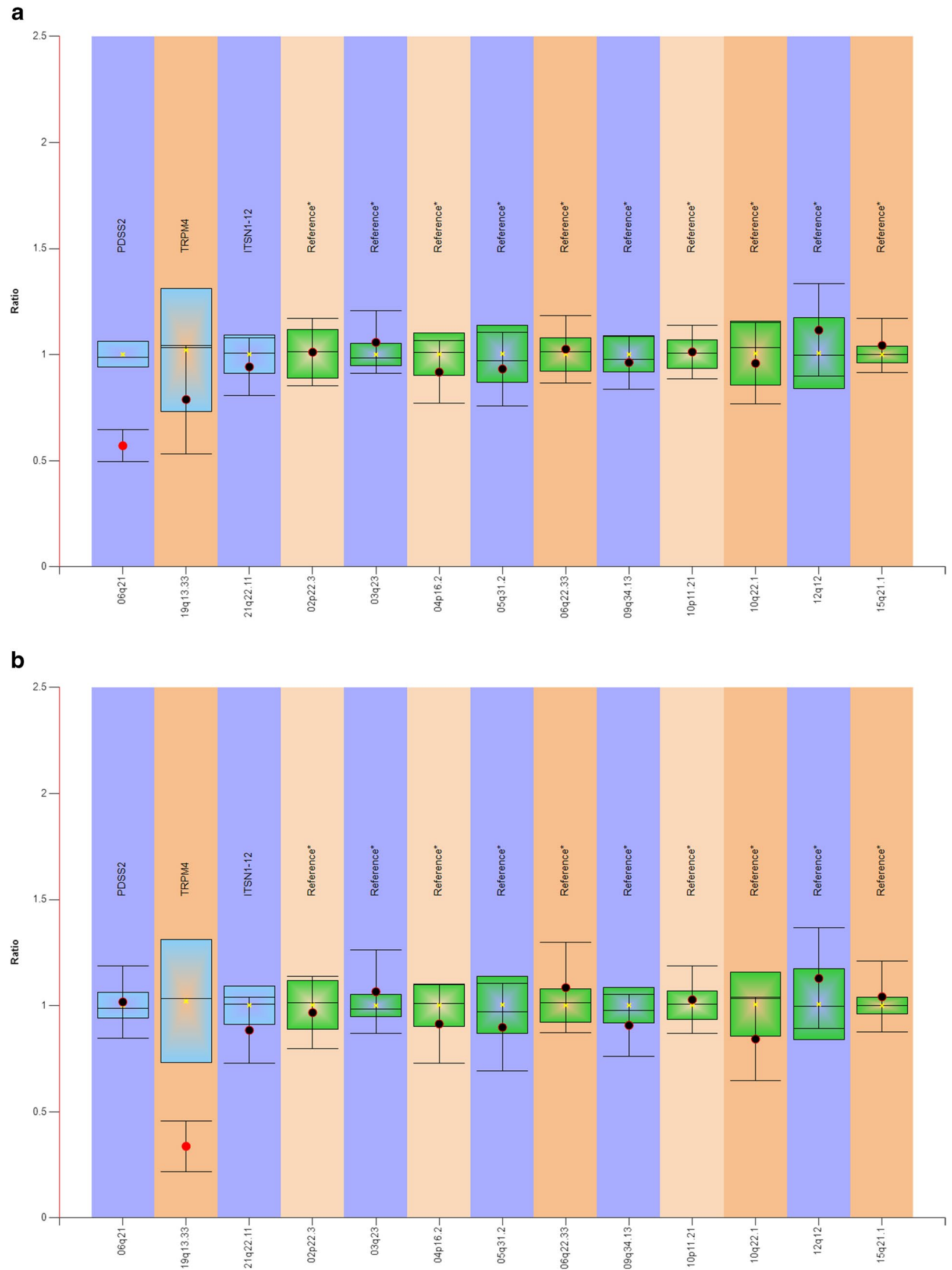

Fig. 1 SVs confirmed by MLPA. Blue/green bars represent 95\% confidence interval over the reference samples $(N=3)$, and dots with lines represent $95 \%$ confidence interval estimate for each probe. In our case, the 95\% confidence intervals of a PDSS2 in SUD058 and b TRPM4 in SUD075 did not overlap, which suggests a heterozygous deletion 
Database of Genomic Variants (DGV) [38], the deletion type was only observed once within 10,847 samples. Such a low frequency could meet the criteria for a pathogenic SV. The protein encoded by PDSS2 is an enzyme that synthesizes the prenyl side chain of coenzyme $\mathrm{Q}_{10}$, which is one of the key elements in the respiratory chain. Previous research has revealed that individuals with primary coenzyme $\mathrm{Q}_{10}$ deficiency could have manifestations associated with multisystemic diseases, including encephalopathy and hypertrophic cardiomyopathy [46]. Therefore, we have reason to suspect SVs that alter the function or expression of this gene might be pathogenic variants. In addition, it is noteworthy that a missense mutation with uncertain significance in $R Y R 2$ (p.(Ala3814Val)) has also been found in this individual. However, it is not clear what the biological effects of these genetic variants are and whether they may have contributed to the cause of death in this young girl.

The other $35 \mathrm{bp}$ heterozygous deletion in TRPM4 was reported to have conflicting interpretations of pathogenicity (benign, likely benign, or uncertain significance) according to the NCBI ClinVar database. As a calcium-activated ion channel encoding gene, TRPM4 had already been extensively studied in a series of channelopathy-related reports, and some uncommon missense SNVs had been identified to be likely pathogenic in 20 out of $248 \mathrm{BrS}$ patients and in 13 out of 330 SUD cases, respectively $[47,48]$. One study had demonstrated in-frame deletions in individuals with cardiac conduction disturbances, but the deletions co-existed with other missense variants, which makes it hard to determine their real functional impact [49]. In our case, beside the heterozygous deletion in TRPM4, a pathogenic stop variant in the gene LZTRI (p. (Gln814*)) was identified. Variants in LZTRI are associated with Noonan syndrome, which is a genetic disorder that causes multiple congenital abnormalities and characteristic facial features that evolve with age [50]. Furthermore, a small portion of patients with Noonan syndrome were reported to show cardiovascular diseases, including atrial septal defects and hypertrophic cardiomyopathy [51].

It is worth noting that a large proportion of SVs identified in this study were located in the intergenic or intronic regions of our target genes and thus were of unknown significance. The evaluation of variants in these noncoding regions has always been challenging as the knowledge about their contribution to electrophysiological dysfunction is still very limited. Introns are usually considered to contribute to the control of gene expression if regulatory regions and noncoding functional RNA genes are affected [52-54]. A recent study combined the most extensive maps of CNVs in human populations and discovered that intronic losses are the most frequent CNVs in protein-coding genes [55]. Therefore, the significance of SVs identified in the intronic regions of our target genes might need to be carefully evaluated by functional studies. Moreover, a recent study has cross-referenced human transcriptome, epigenomic, and chromatin datasets to find causal genetic variants in noncoding regions that alter the functionality of transcription regulatory elements and target gene expression associated with atrial fibrillation (AF) [56]. With an improved ability to identify these genetic variants neglected by most previous studies, the pathogenic mechanism behind SUD might eventually be better explained by routine SNV and SV testing in suspected SUD cases.

There are some limitations in our current study. Since we only focused on 244 cardiac-related genes, variants outside these regions could not be identified. Besides, SVs longer than $1 \mathrm{Mbp}$ were not included in our candidate list due to the difficulties in confirming the MPS result. In addition, functional studies would be required to further investigate the 2 identified SVs in PDSS2 and TRPM4 in order to verify their potential pathogenic role and contribution to the sudden death event of these 2 SUD cases. When several potential pathogenic variants are under consideration, it will be important to find out which of the detected variants contributed most to the sudden unexpected death event. In our study, family members were not available for co-segregation analyses. This would be necessary to determine the mode of inheritance and to identify other family members at risk for sudden cardiac death.

In conclusion, our study supports that SVs in cardiac disease-associated genes might be involved in some SUD cases. However, the functional interpretation of pathogenic SVs is complex and genetic evidence should be used cautiously in molecular diagnosis.

Supplementary Information The online version contains supplementary material available at https://doi.org/10.1007/s00414-021-02580-5.

Acknowledgment The authors would like to thank Dr. Urs Graf and Alessandro Maspoli from the Institute of Medical Molecular Genetics (University of Zurich, Switzerland) for their help in data interpretation of the exome data. The authors express their gratitude to the Emma Louise Kessler Foundation that supported this study.

Funding Open Access funding provided by University of Zurich. The study was funded by the Emma Louise Kessler Foundation. The exome analysis was supported by the Swiss National Science Foundation (SNF, project-No. 320030-149456, 2013-2017). Shouyu Wang was financially supported by the fund of China Scholarship Council (Nr. 201906240236).

\section{Declarations}

Ethics approval Ethical approval for this study was provided by the local ethics committee in Zurich (KEK-ZH-Nr. 2013-0086), and the study was conducted in full conformance with Swiss laws and regulations. The requirements of the local ethics committee included written informed consent of family members. If no family members were available, SUD cases had been irreversibly anonymized. 
Conflicts of interest The authors declare no competing interests.

Open Access This article is licensed under a Creative Commons Attribution 4.0 International License, which permits use, sharing, adaptation, distribution and reproduction in any medium or format, as long as you give appropriate credit to the original author(s) and the source, provide a link to the Creative Commons licence, and indicate if changes were made. The images or other third party material in this article are included in the article's Creative Commons licence, unless indicated otherwise in a credit line to the material. If material is not included in the article's Creative Commons licence and your intended use is not permitted by statutory regulation or exceeds the permitted use, you will need to obtain permission directly from the copyright holder. To view a copy of this licence, visit http://creativecommons.org/licenses/by/4.0/.

\section{References}

1. Ackerman MJ et al (2011) HRS/EHRA expert consensus statement on the state of genetic testing for the channelopathies and cardiomyopathies: this document was developed as a partnership between the Heart Rhythm Society (HRS) and the European Heart Rhythm Association (EHRA). Europace 13(8):1077-1109. https:// doi.org/10.1093/europace/eur245

2. Markwerth P et al (2020) Sudden cardiac death-update. Int J Legal Med. https://doi.org/10.1007/s00414-020-02481-z

3. Narula $\mathrm{N}$ et al (2014) Post-mortem whole exome sequencing with gene-specific analysis for autopsy-negative sudden unexplained death in the young: a case series. Pediatr Cardiol 36(4):768-778. https://doi.org/10.1007/s00246-014-1082-4

4. Neubauer J et al (2018) Exome analysis in 34 sudden unexplained death (SUD) victims mainly identified variants in channelopathyassociated genes. Int J Legal Med 132(4):1057-1065. https://doi. org/10.1007/s00414-018-1775-y

5. Anderson JH et al (2016) Whole-exome molecular autopsy after exertion-related sudden unexplained death in the young. Circ Cardiovasc Genet 9(3):260-265. https://doi.org/10.1161/CIRCG ENETICS.115.001370

6. Christiansen SL et al (2016) Genetic investigation of 100 heart genes in sudden unexplained death victims in a forensic setting. Eur J Hum Genet 24(12):1797-1802. https://doi.org/10.1038/ejhg. 2016.118

7. Bezzina Connie R et al (2006) Common sodium channel promoter haplotype in Asian subjects underlies variability in cardiac conduction. Circulation 113(3):338-344. https://doi.org/10.1161/ CIRCULATIONAHA.105.580811

8. Park JK et al (2012) Genetic variants in SCN5A promoter are associated with arrhythmia phenotype severity in patients with heterozygous loss-of-function mutation. Heart Rhythm 9(7):1090-1096. https://doi.org/10.1016/j.hrthm.2012.02.023

9. Zhao Y et al (2015) Post-transcriptional regulation of cardiac sodium channel gene SCN5A expression and function by miR192-5p. Biochim Biophys Acta 1852(10 Pt A):2024-2034

10. Wang $S$ et al (2017) An insertion/deletion polymorphism within 3'UTR of RYR2 modulates sudden unexplained death risk in Chinese populations. Forensic Sci Int 270:165-172. https://doi.org/ 10.1016/j.forsciint.2016.12.005

11. Sonoda $\mathrm{K}$ et al (2018) Copy number variations of SCN5A in Brugada syndrome. Heart Rhythm. https://doi.org/10.1016/j.hrthm. 2018.03 .033

12. Li Mura IE et al (2013) Identification of a PKP2 gene deletion in a family with arrhythmogenic right ventricular cardiomyopathy.
Eur J Hum Genet 21(11):1226-1231. https://doi.org/10.1038/ ejhg.2013.39

13. Mates J et al (2020) Sudden cardiac death and copy number variants: what do we know after 10 years of genetic analysis? FSI:Genetics 47. https://doi.org/10.1016/j.fsigen.2020.102281

14. Tester DJ et al (2020) Identification of a novel homozygous multi-exon duplication in RYR2 among children with exertionrelated unexplained sudden deaths in the Amish community. JAMA Cardiol. https://doi.org/10.1001/jamacardio.2019.5400

15. Layer RM et al (2014) LUMPY: a probabilistic framework for structural variant discovery. Genome Biol 15(6):R84. https:// doi.org/10.1186/gb-2014-15-6-r84

16. Sharp AJ et al (2005) Segmental duplications and copy-number variation in the human genome. The American Journal of Human Genetics 77(1):78-88. https://doi.org/10.1086/431652

17. Redon $\mathrm{R}$ et al (2006) Global variation in copy number in the human genome. Nature 444(7118):444-454. https://doi.org/10. 1038 /nature 05329

18. Nakajima $\mathrm{T}$ et al (2008) HIV-1/AIDS susceptibility and copy number variation in CCL3L1, a gene encoding a natural ligand for HIV-1 co-receptor CCR5. Cytogenet Genome Res 123(14):156-160. https://doi.org/10.1159/000184703

19. Glessner JT et al (2009) Autism genome-wide copy number variation reveals ubiquitin and neuronal genes. Nature 459(7246):569-573. https://doi.org/10.1038/nature07953

20. Glessner JT et al (2010) Strong synaptic transmission impact by copy number variations in schizophrenia. Proc Natl Acad Sci U S A 107(23):10584-10589. https://doi.org/10.1073/pnas.10002 74107

21. Glessner JT et al (2010) Duplication of the SLIT3 locus on 5 q35.1 predisposes to major depressive disorder. PLoS One 5(12):e15463. https://doi.org/10.1371/journal.pone.0015463

22. Mates $\mathbf{J}$ et al (2018) Role of copy number variants in sudden cardiac death and related diseases: genetic analysis and translation into clinical practice. Eur J Hum Genet. https://doi.org/10. 1038/s41431-018-0119-1

23. Andersen JD et al (2019) Whole genome and transcriptome sequencing of post-mortem cardiac tissues from sudden cardiac death victims identifies a gene regulatory variant in NEXN. Int J Legal Med 133(6):1699-1709. https://doi.org/10.1007/ s00414-019-02127-9

24. Tester DJ et al (2020) Identification of a novel homozygous multi-exon duplication in RYR2 among children with exertionrelated unexplained sudden deaths in the Amish community. JAMA Cardiology 5(3):13-18. https://doi.org/10.1001/jamac ardio. 2019.5400

25. Jaouadi $\mathrm{H}$ et al (2020) Multiallelic rare variants support an oligogenic origin of sudden cardiac death in the young. Herz. https://doi.org/10.1007/s00059-019-04883-1

26. Richards $\mathrm{S}$ et al (2015) Standards and guidelines for the interpretation of sequence variants: a joint consensus recommendation of the American College of Medical Genetics and Genomics and the Association for Molecular Pathology. Genet Med 17(5):405-424. https://doi.org/10.1038/gim.2015.30

27. Sabatasso $\mathrm{S}$ et al (2020) Second opinion system for sudden cardiac death cases in forensic practice. Int J Legal Med. https:// doi.org/10.1007/s00414-019-02225-8

28. Medeiros Domingo A et al (2018) Recommendations for genetic testing and counselling after sudden cardiac death: practical aspects for Swiss practice. Swiss Med Wkly 148:w14638. https://doi.org/10.4414/smw.2018.14638

29. Fellmann F et al (2019) European recommendations integrating genetic testing into multidisciplinary management of sudden cardiac death. Eur J Hum Genet 27(12):1763-1773. https://doi. org/10.1038/s41431-019-0445-y 
30. Li H, Durbin R (2010) Fast and accurate long-read alignment with Burrows-Wheeler transform. Bioinformatics 26(5):589-595. https://doi.org/10.1093/bioinformatics/btp698

31. McKenna A et al (2010) The genome analysis toolkit: a MapReduce framework for analyzing next-generation DNA sequencing data. Genome Res 20(9):1297-1303. https://doi.org/10.1101/gr. 107524.110

32. DePristo MA et al (2011) A framework for variation discovery and genotyping using next-generation DNA sequencing data. Nat Genet 43(5):491-498. https://doi.org/10.1038/ng.806

33. Robinson JT et al (2011) Integrative genomics viewer. Nat Biotechnol 29(1):24-26. https://doi.org/10.1038/nbt.1754

34. Wang Q et al (2020) Landscape of multi-nucleotide variants in 125,748 human exomes and 15,708 genomes. Nat Commun 11(1):2539. https://doi.org/10.1038/s41467-019-12438-5

35. Kopanos C et al (2019) VarSome: the human genomic variant search engine. Bioinformatics 35(11):1978-1980. https://doi.org/ 10.1093/bioinformatics/bty897

36. Li H et al (2009) The Sequence Alignment/Map format and SAMtools. Bioinformatics 25(16):2078-2079. https://doi.org/10.1093/ bioinformatics/btp352

37. Wang K et al (2010) ANNOVAR: functional annotation of genetic variants from high-throughput sequencing data. Nucleic Acids Res 38(16):e164. https://doi.org/10.1093/nar/gkq603

38. MacDonald JR et al (2014) The Database of Genomic Variants: a curated collection of structural variation in the human genome. Nucleic Acids Res 42(Database issue):D986-D992. https://doi. org/10.1093/nar/gkt958

39. Collins RL et al (2020) A structural variation reference for medical and population genetics. Nature 581(7809):444-451. https:// doi.org/10.1038/s41586-020-2287-8

40. Zeek PM (1942) Heart weight I. The weight of the normal human heart. Arch Pathol 34:820-832

41. Arbustini E et al (2016) Left ventricular noncompaction: a distinct genetic cardiomyopathy? J Am Coll Cardiol 68(9):949-966. https://doi.org/10.1016/j.jacc.2016.05.096

42. Li W et al (2018) SCN5A variants: association with cardiac disorders. Front Physiol 9:1372. https://doi.org/10.3389/fphys.2018. 01372

43. Hancox JC et al. (2017) Triadin mutations - a cause of ventricular arrhythmias in children and young adults. J Congenital Cardiol 1(1). https://doi.org/10.1186/s40949-017-0011-9

44. Campuzano O et al (2020) Reanalysis and reclassification of rare genetic variants associated with inherited arrhythmogenic syndromes. EBio Med 54:102732. https://doi.org/10.1016/j. ebiom.2020.102732

45. Tester DJ et al (2018) Cardiac Genetic Predisposition in Sudden Infant Death Syndrome. J Am Coll Cardiol 71(11):1217-1227. https://doi.org/10.1016/j.jacc.2018.01.030

46. Salviati L et al (2017) Primary coenzyme $\mathrm{Q}_{10}$ deficiency. In: Gene reviews [Internet]. Seattle (WA): University of Washington, Seattle, 28125198

47. Liu $\mathrm{H}$ et al (2013) Molecular genetics and functional anomalies in a series of 248 Brugada cases with 11 mutations in the TRPM4 channel. PLoS ONE 8(1):e54131-e54131. https://doi.org/10. 1371/journal.pone.0054131

48. Subbotina E et al (2018) Functional characterization of TRPM4 variants identified in sudden unexpected natural death. Forensic Sci Int 293:37-46. https://doi.org/10.1016/j.forsciint.2018.10.006

49. Stallmeyer B et al (2012) Mutational spectrum in the Ca2+activated cation channel gene TRPM4 in patients with cardiac conductance disturbances. Hum Mutat 33(1):109-117. https://doi. org/10.1002/humu.21599

50. Romano AA et al (2010) Noonan syndrome: clinical features, diagnosis, and management guidelines. Pediatrics 126(4):746759. https://doi.org/10.1542/peds.2009-3207

51. Prendiville TW et al (2014) Cardiovascular disease in Noonan syndrome. Arch Dis Child 99(7):629-634. https://doi.org/10. 1136/archdischild-2013-305047

52. Rose AB (2008) Intron-Mediated Regulation of Gene Expression. In: Reddy ASN, Golovkin M (eds) Nuclear pre-mRNA Processing in Plants. Springer Berlin Heidelberg, Berlin, pp 277-290. https:// doi.org/10.1007/978-3-540-76776-3_15

53. Le Hir $\mathrm{H}$ et al (2003) How introns influence and enhance eukaryotic gene expression. Trends Biochem Sci 28(4):215-220. https:// doi.org/10.1016/S0968-0004(03)00052-5

54. Jo B-S, Choi SS (2015) Introns: the functional benefits of introns in genomes. Genomics Inform 13(4):112-118. https://doi.org/10. 5808/GI.2015.13.4.112

55. Rigau $\mathrm{M}$ et al (2019) Intronic $\mathrm{CNVs}$ and gene expression variation in human populations. PLoS Genet 15(1):e1007902-e1007902. https://doi.org/10.1371/journal.pgen.1007902

56. van Ouwerkerk AF et al (2019) Identification of atrial fibrillation associated genes and functional non-coding variants. Nat Commun 10(1):4755-4755. https://doi.org/10.1038/s41467-019-12721-5

Publisher's note Springer Nature remains neutral with regard to jurisdictional claims in published maps and institutional affiliations. 